\title{
Study on the Technology of Surface Hardening of Steel by Electrical Discharge Machining
}

\author{
Yang Li \\ Tianjin University of Technology and Education \\ Tianjin 300222, PR China
}

\begin{abstract}
In this paper, by electrospark surface strengthening of $\mathrm{H} 13$ steel and AL powder mixed near dry fog in themedium, is made of titanium alloy electrode, and the microstructure of hardened layer, thickness and hardness were analyzed. The analysis results show that the obtained in 3 different working medium under the condition of strengthening layer are with orange peel, and the strengthening layer section from the outside to the inside followed by white layer composition, transition layer and matrix. The strengthening layer surface is smoother in AL powder mixed near dry medium conditions, more compact structure, and the coating's thickness is higher than kerosene fog and working medium under the condition of thickness distribution is more uniform. The hardness of strengthened layer compared with the substrate. The average microhardness is higher than that of mist and kerosene medium under the condition of quasi dry working medium of $\mathrm{Al}$ powder .
\end{abstract}

Keywords: H13 steel; Electric Spark Surface Strengthening; Powder Mixing Quasi Dry; Microstructure; Microhardness

\section{INTRODUCTION}

H13 steel is a kind of hot working die steel which is widely used in the world. It has high hardenability and hot cracking resistance. [1. The surface of the mould is usually first damaged, so it is necessary to strengthen the surface of the mould to prolong the service time. [2]. In recent years, researchers have carried out extensive research on various surface strengthening technologies of H13 steel, such as traditional chemical heat treatment surface Carburizing or nitrogen, laser cladding, micro-arc oxidation, ion implantation and so on. However, such as high heating temperature, long heat treatment time, thin infiltrating layer, uneven surface of alloy, difficult control of composition and microstructure, bonding of reinforcing layer with matrix The surface layer of hard layer is loose , easy to fall off and equipment is expensive

The EDM strengthening technique is by the transient discharge between the electrode and the matrix, the electrode material diffuses and migrates to the matrix by using the discharge energy, in order to realize the partial surface melting and alloying of the matrix, the hard thin layer compound on the surface can be obtained. [In order to improve the surface mechanical properties of the strengthened workpiece. Traditional EDM surface strengthening is mostly carried out in a single medium with certain insulating properties [5-11]. Furutani and Wang Zhenlong and others have made research and found that:. Surface strengthening with liquid as the medium changed the surface characteristics of the material. Improved microhardness, wear resistance and corrosion resistance. The working liquid gasification and expansion of melting to the electrode surface strengthening material erosion

\author{
Guangxing Sun \\ Tianjin University of Technology and Education \\ Tianjin 300222, PR China
}

is very strong, so it is not conducive to strengthening layer, strengthening layer thickness of thin 5-6. Zhang Ming using YK10 hard alloy rods as the tool electrode, M2 high speed steel workpiece EDM surface strengthening in the air in the enhancement layer and the substrate tightly, its hardness is between cemented carbide and high speed steel. The hardness of [7 with air as the working medium can expand the scope of application of electrospark surface strengthening, but the existing machining accuracy uniformity, strengthening thin layer deficiency. The fog (gas-liquid mixing) as the working medium by the first Japanese scholar T In this paper, the advantages of better cooling function, low manufacturing cost and low electrode manufacturing cost are compared with that of gas medium discharge machining by using gas medium . The microstructure and microhardness of the reinforced layer under different working medium conditions are studied .

\section{EXPERIMENT}

H13 steel, commonly known as 4Cr5MoVSiin our country, is a new type of hot working die steel which is widely used in the world. The chemical composition of H13 steel is shown in Table 1.

Table 1 Chemical Composition of Steel 13 Steel

\begin{tabular}{|c|c|c|c|c|c|c|c|c|}
\hline $\begin{array}{l}\text { ele } \\
\text { ment }\end{array}$ & $\mathrm{C}$ & $\mathrm{Si}$ & $\mathrm{Mn}$ & $\mathrm{Cr}$ & Mo & V & $S$ & $\mathrm{P}$ \\
\hline \multirow{5}{*}{$\%$} & 0 & 0 & 0 & 4 & 1 & 0 & & \\
\hline & .32 & .80 & .20 & .75 & .10 & .80 & $\leqslant$ & $\leqslant$ \\
\hline & $\sim$ & $\sim$ & $\sim$ & $\sim$ & $\sim$ & $\sim$ & 0.30 & 0.30 \\
\hline & 0 & 1 & 0 & 5 & 1 & 1 & 0 & 0 \\
\hline & .45 & .20 & .50 & .50 & .75 & .20 & & \\
\hline
\end{tabular}

After corrosion of the $3 \%$ nitric acid alcohol etchant for the raw sample of the steel 13 steel, it was observed that the microstructure had a granular pearlite and a lamellar pearlite ( very few ) at a multiple of $500 \mathrm{x}$, as shown in FIG . 1 with a hardness of about $350 \mathrm{HB}$

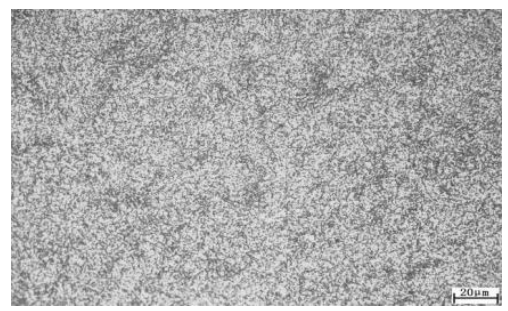

Fig . 1 The original structure of steel 13 steel

An electric spark surface strengthening steel 13 steel test was carried out using an electric spark forming machine tool ( AF1100 ), which was a tool electrode of titanium alloy, and 
was carried out under the condition of kerosene, fog ( mixture of air and deionized water ) and semi - dry ( air , deionized water and $\mathrm{AL}$ powder ) as working medium .

The electrical parameters of EDM surface strengthening were: pulse current $\mathrm{Ie}=20.8 \mathrm{~A}$, pulse width ton $=50 \mathrm{us,}$, pulse interval 50 us. machining depth $0.2 \mathrm{~mm}$. The working polarity is positive (the workpiece is connected with the positive pole of the power supply and the tool electrode is connected with the negative electrode of the power supply). The size of the sample used in the EDM surface strengthening test is $10 \mathrm{~mm}$ $\times 10 \mathrm{~mm} \times 3 \mathrm{~mm}$. After grinding and polishing of $180-600$ metallographic sandpaper, the surface strengthening test of EDM was carried out. The microstructure of the original alloy and the surface of the strengthened layer was observed under scanning electron microscope (VEGAII SBH). In Wilso The microhardness test was carried out under the condition of micro - Vickers hardness of 402MVD, the selection load was $0.45 \mathrm{~N}$, the loading time was $10 \mathrm{~s}, 3$ different points were randomly tested for each sample, and the hardness average value was obtained.

\section{ETEST RESULTS AND ANALYSIS}

\section{Microstructure and morphology of strengthened layers}

in that condition of kerosene medium , the microstructure of the reinforce layer on the surface of the strengthened layer is in the orange peel shape, the edge of the orange peel - like tissue is uniform, the distribution of the reinforce layer is uniform , the distribution of the reinforcing layer is uniform , the distribution of the reinforcing layer is uniform, the surface quality of the reinforcing layer is more uniform, and a small amount of micro - crack is present. In addition to spatter, there are no cracks, and the overall strengthening effect is better. From fig. 2, it can be seen that the single pulse strengthening point of $\mathrm{H} 13$ steel matrix is round under three different working medium conditions, and there is a circular pit in the center. This is due to the "gas explosion" produced by the instantaneous high energy pulse of electric spark discharge resulting in droplet splashing of the alloy [12].

For ease of comparison, the simulation results based on the predictive theory of this paper are also added to the graph. It can be seen from the figure that the model results predict the results of the cutting force and the test results, whether the cutting force curve cycle, waveform or amplitude are very good consistency.
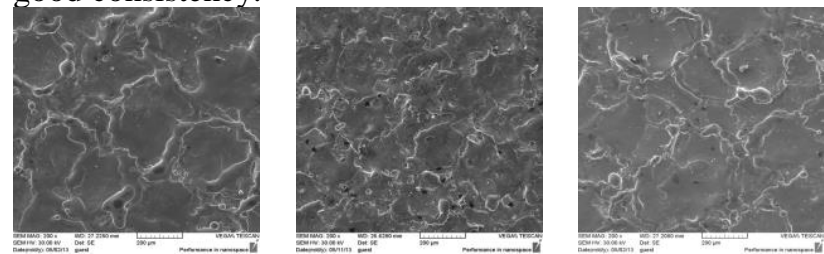

(a)Kerosene medium (b)Fog medium(c)Powder mixed near dry medium

Figure 2 :microstructure of $\mathrm{H} 13$ steel after intensification of different medium surface

\section{Micro-tissue morphology of reinforced layer section}

Figure 3 is a section of strengthened layer SEM images obtained under different working medium. Figure 3 (a) in kerosene enhanced level thickness was significantly thinner, the maximum thickness of the 15.10um and the distribution is not uniform, this is because the oil constantly scouring the surface of the workpiece, the electrical erosion will have thrown and removed. The strengthening layer thickness is thin is not conducive to strengthening layer formation; Figure 3 (b) enhanced layer thickness was obviously thicker in the mist medium, the maximum thickness can reach 18.63um, and the distribution is more uniform. Figure 3 (c) in AL powder mixed near dry medium, strengthening layer is more obvious than the fog and kerosene medium The thickness of the underlayer is higher, the thickness of the strengthened layer is as high as 23.14um, and the distribution is more uniform, which is due to the fog and mixed AL powder quasi dry medium. The fog formed by the mixture of gas and droplet will not cause the effect of scouring the workpiece surface. At the same time the droplet is vaporized by the plasma and the volume will increase more than 1000 times which can provide the explosive force of the discharge channel for the working medium. The cooling effect is better. [13], all of these are favorable to the formation of the strengthening layer and increase the thickness of the strengthening layer. At the same time, the combination of the strengthening layer and the matrix is better under these two media conditions, and there is no obvious boundary layer. The internal structure of the strengthened layer is uniform and dense, and strong. Followed by the section layer from the outside to the inside the white layer, transition layer and matrix composition, this is because the electrospark surface strengthening is high temperature application of electric spark discharge, the substrate material and the electrode material on the substrate surface melting occurs in situ reaction for the formation of a secondary growth
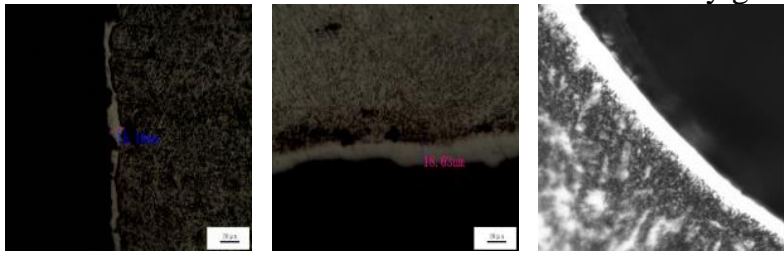

(a)Kerosene medium(b)Fog medium(c)Mixed AL powder quasi dry medium

Fig.3 SEM image of reinforced layer under different media conditions

\section{Hardness test of reinforcement layer}

The microhardness was tested by Wilson microhardness tester $402 \mathrm{MVD}$. The selected load was $0.45 \mathrm{~N}$ and the loading time was 10s. The microhardness of each sample was tested at 3 different points at random, and the average hardness was obtained. The test data can be seen in Table 2.Compared with the original $\mathrm{H} 13$ steel, it can be obtained from Table 2. After surface strengthening of EDM in three different medium conditions, the surface hardness of EDM was greatly improved. The average hardness of the strengthened layer in kerosene medium was $843.7 \mathrm{HV}$. The average hardness of the strengthened layer was $911.7 \mathrm{HV}$ in fog medium, while in the mixed AL powder quasi dry medium, the hardness of the strengthened layer was $911.7 \mathrm{HV}$. The average hardness of the strengthened layer is up to $1004 \mathrm{HV}$. it can be seen from above that the hardness of mixed AL powder in quasi-dry medium is obviously higher than that in kerosene and fog medium.

Microhardness of enhanced layer (HV) obtained in Table 2 under different working medium conditions 


\section{CONCLUSION}

(1)In the mist of kerosene, and AL powder mixed near dry medium under the condition of the H13 steel test electric spark strengthening, strengthening the overall morphology of tissue layer showed orange peel, in AL powder mixed near dry medium under the condition of strengthening layer surface quality is better, in addition to occasional splash, no cracks, overall strengthening effect good.

(2)Three different working conditions, AL powder mixed near dry strengthening layer and the substrate of the best, and strengthen the greater thickness distribution is more uniform.

( 3 ) The hardness of the strengthened layer was greatly improved after electric spark surface strengthening experiment under different working medium conditions, and the average microhardness of the strengthened layer under quasi dry working medium of the mixed AL powder was obviously higher than that of the reinforcing layer under the conditions

\begin{tabular}{lllll}
\hline $\begin{array}{c}\text { ambient } \\
\text { condition }\end{array}$ & Point 1 & Point 2 & Point 3 & $\begin{array}{l}\text { average } \\
\text { value }\end{array}$ \\
\hline kerosene & 876 & 901 & 754 & 843.7 \\
fog & 948 & 867 & 920 & 911.7 \\
$\begin{array}{c}\text { Mixedquasi } \\
\text { dry powder }\end{array}$ & 1037 & 962 & 1014 & 1004 \\
\hline
\end{tabular}

of mist and kerosene medium .

\section{REFERENCES:}

[1] Wang Peng, Zhang Jijiang and so on . In this paper, the application status quo of steel 13 steel is described in detail . Die Manufacturing , 2007 ( 12 ) : 1 - 7

[2] He Berlin.Mould Materials and Surface strengthening Technology. Beijing Chemical Industry Press, China (in Chinese).

[3] Zhang Chunhui, Ma Hongyan, Wang Maocai . New progress on surface strengthening of titanium alloys Progress in titanium alloys industry , 2003, 20 ( 4 / 5 ): 49 - 52

[4] Tang smart, Qiao Shengru. Two kinds of steel reinforcement layer structure of $[\mathrm{J}]$. thermal processing technology of EDM surface materials, 2006,35 (20): 19-21.

[5] Mohri N,Saito N,TSunekawa Y. Metal surface modification byelectrical discharge machining with composite electrodes [J]. Annals of the CIRP. 1993,42(1):219-222.
[6] Furutani K,Saneto A,Takezawa H,et.al. Accretion of titanium carbide byelectrical discharge machining with powder suspended in working fluid. Journal of the International Societies for Precision En Gineering and Nanotechnology [J].2001,25:138 144 .

[7] Zhang Ming, Li Fujie. Experimental research on discharge deposition in air of EDM machine tool. [J]. tool technology. 2009,43 (6):40 142.

[8] [8] TANIMURA T,ISUZUGAW K,JITAI. Etal.Develop-ment of EDM in the mst [J] .Proc.ISEM,1989(9): 313-316.

[9] L.Gu, W.S.Zhao, L.Li, Y.G Xia. K.P.Rajurkar. Electrical DischargeMachininginJettedMist[C].ISEMXV,Pittsburgh,USA, 2007.Nebraska: Industrial and Management Systems Engineering,2007:297-300

[10] Zhao Wansheng, Zhao Wansheng, Zhang Zhihua , Kang Xiaoming . Analysis of machining and mechanism of spray electric spark milling . Electric machining and mould . 2006,2 $1-4$

[11] Li Li, Gu Lin, Xia Yonggao, Zhao Wansheng. Experimental research on the internal spray type electric spark milling $[\mathrm{J}]$. Journal of Shanghai Jiao Tong University.2007,41 (10): 15691. 1572

[12] Bai Xue, Zhang Qinghe, Li Tian Tian, et al.Study on breakdown mechanism of mixed powder quasi dry EDM medium. [J] .Acta mechatronica Sinica: 186-192.

[13] Kunieda M. Furuoya S. Improvement of EMD efficiency by sup-plying oxygen gas. Into gap [J]. Annals of the CIRP. $1991,40(1): 215-218$. 